\title{
CHEBYSHEV APPROXIMATION BY FAMILIES WITH THE BETWEENESS PROPERTY
}

BY

CHARLES B. DUNHAM

1. Introduction. In this note a theory of Chebyshev approximation is obtained for approximating families with a property which is a generalization of convexity, the betweeness property. This theory is of interest for several reasons. Most of the approximating families for which a tractable theory exists characterize best approximations by the extrema of their error curve. The betweeness property is the weakest easily verifiable condition giving such a characterization of best approximations. The development of the theory sheds considerable light on the well-known linear theory [2], [5] and rational theory [1], [2], [3]. A necessary and sufficient condition for the uniqueness of best approximations is obtained; it is the most general known necessary and sufficient condition for any theory.

Let $X$ be a compact space and for a function $g$ define $\|g\|=\sup \{|g(x)|: x \in X\}$.

Let $\mathscr{G}$ be a family of real continuous functions with elements $F, G, H, \ldots$ The Chebyshev problem is: given a continuous function $f$, to find an element $G^{*}$ of $\mathscr{G}$ to minimize $e(G)=\|E(G, \cdot)\|$ where $E(G, x)=f(x)-G(x)$. Such an element $G^{*}$ is called a best approximation in $\mathscr{G}$ to $f$ on $X$. It will be assumed throughout the discussion that $f$ is fixed, and mention of $f$ is suppressed in the notation $e(G)$ and $E(G, \cdot)$.

The author wishes to thank Professor E. Barbeau for his careful criticism of the hypotheses and proofs of this note.

\section{The betweeness property.}

Definition. A family $\mathscr{G}$ of real continuous functions is said to have the $b e$ tweeness property if for any two elements $G_{0}$ and $G_{1}$, there exists a $\lambda$-set $\left\{H_{\lambda}\right\}$ of elements of $\mathscr{G}$ such that $H_{0}=G_{0}, H_{1}=G_{1}$, and for all $x \in X, H_{\lambda}(x)$ is either a strictly monotonic continuous function of $\lambda$ or a constant, $0 \leqq \lambda \leqq 1$. (It should be noted that $H_{\lambda}(x)$ can be monotone in different senses for different $x$.)

LEMMA 1. Let $\left\{G_{k}\right\}$ be a sequence of continuous functions on a compact space $X$ such that $\left\{G_{k}\right\}$ converges pointwise to a continuous function $G_{0}$ and for any $x \in X$, $G_{k}(x)$ is a monotonic sequence, then $\left\{G_{k}\right\}$ converges uniformly to $G_{0}$.

Proof. The sequence $\left|G_{k}(x)-G_{0}(x)\right|$ is a decreasing sequence of continuous functions, which converges to the continuous limit 0 . By Dini's theorem, the convergence is uniform. From this lemma it can be seen that if $\left\{H_{\lambda}\right\}$ is a $\lambda$-set for $G_{0}$ and $G_{1}$ then the sequence $\left\{H_{1 / k}\right\}$ converges uniformly to $G_{0}$.

Received by the editors February 17, 1967 and, in revised form, October 12, 1967. 
Any linear family $\mathscr{L}$ of continuous functions (and any convex subset of $\mathscr{L}$ ) has the betweeness property, for a $\lambda$-set is given by $H_{\lambda}=\lambda G_{1}+(1-\lambda) G_{0}$.

More generally let $\mathscr{P}$ and $\mathscr{Q}$ be linear families and $\mathscr{F}$ a convex set of pairs $(p, q), p \in \mathscr{P}, q \in \mathscr{Q}$ such that $(p, q) \in \mathscr{F}$ implies $(\alpha p, \alpha q) \in \mathscr{F}$ for $\alpha>0$. A function is an $\mathscr{F}$-admissible rational function if it is of the form $p / q,(p, q) \in \mathscr{F}, q>0$. The set $\mathscr{R}(\mathscr{F})$ of $\mathscr{F}$-admissible rational functions has the betweeness property, for the $\lambda$-set corresponding to $p_{0} / q_{0}$ and $p_{1} / q_{1}$ is

$$
\begin{aligned}
H_{\lambda} & =\left(\lambda p_{1}+(1-\lambda) p_{0}\right) /\left(\lambda q_{1}+(1-\lambda) q_{0}\right), \\
d H_{\lambda} / d \lambda & =\left(p_{1} q_{0}-p_{0} q_{1}\right) /\left(\lambda q_{1}+(1-\lambda) q_{0}\right)^{2}
\end{aligned}
$$

being of constant sign for a given point $x$ and vanishing identically at any point $x$ at which $p_{0} / q_{0}-p_{1} / q_{1}$ vanishes. In the case where $\mathscr{F}$ consists of all pairs we obtain the family

$$
\mathscr{R}=\{p / q: p \in \mathscr{P}, q \in \mathscr{Q}, q>0\}
$$

of admissible rational functions.

If $\phi$ is a continuous strictly monotonic function from the real line into the real line and $\mathscr{G}$ has the betweeness property, then the set of elements of the form $\phi(G)$, $G \in \mathscr{G}$ has the betweeness property, for if $\left\{H_{\lambda}\right\}$ is a $\lambda$-set for $G_{0}$ and $G_{1},\left\{\phi\left(H_{\lambda}\right)\right\}$ is a $\lambda$-set for $\phi\left(G_{0}\right)$ and $\phi\left(G_{1}\right)$.

After the theory of this paper had been obtained it was noticed that Meinardus and Schwedt had used a condition [4, p. 304] quite close to the betweeness property, but developed a different type of theory.

3. Characterization of best approximation. The points at which $E(G, \cdot)$ attains its norm $e(G)$ will be denoted by $M(G)$. By compactness of $X$ and continuity of $E(G, \cdot), M(G)$ is nonempty and closed.

THEOREM 1. Let $\mathscr{G}$ have the betweeness property. An element $G_{0}$ of $\mathscr{G}$ is a best approximation if and only if there exists no element $G_{1} \in \mathscr{G}$ such that $\left|E\left(G_{1}, x\right)\right|$ $<e\left(G_{0}\right)$ for all $x \in M\left(G_{0}\right)$.

Proof. The condition is obviously sufficient for $G_{0}$ to be a best approximation (we do not need the betweeness property). We now prove necessity. Let us suppose that $\left|E\left(G_{1}, x\right)\right|<e\left(G_{0}\right)$ for all $x \in M\left(G_{0}\right)$ then by continuity of $E\left(G_{1}, \cdot\right)$ there exists an open cover $U$ of $M\left(G_{0}\right)$ on which this inequality holds. Let $V=X \sim U$, then if $V$ is empty it is immediate that $G_{0}$ is not best. We therefore suppose that $V$ is nonempty. Let $H_{\lambda}$ be a $\lambda$-set corresponding to $G_{0}$ and $G_{1}, H_{0}=G_{0}, H_{1}=G_{1}$. On the set $U$ we have $E\left(H_{\lambda}, x\right)$ on the open interval between $E\left(G_{0}, x\right)$ and $E\left(G_{1}, x\right)$ for $0<\lambda<1$, hence

$$
E\left(H_{\lambda}, x\right)<e\left(G_{0}\right), \quad 0<\lambda<1, x \in U .
$$

Let $\eta=e\left(G_{0}\right)-\sup \left\{\left|E\left(G_{0}, x\right)\right|: x \in V\right\}$. As $V$ is compact and $E\left(G_{0}, \cdot\right)$ is continuous, $E\left(G_{0}, \cdot\right)$ attains its supremum on $V$ and this supremum cannot be $e\left(G_{0}\right)$, as 
$M(G) \cap V$ is empty, hence $\eta>0$. The sequence $\left\{H_{1 / k}\right\}$ converges uniformly to $G_{0}$. Choose $\delta>0$ such that $\left\|G_{0}-H_{\delta}\right\|<\eta$. It follows that for $x \in V$,

$$
\begin{aligned}
\left|E\left(H_{\delta}, x\right)\right|=\left|f(x)-H_{\delta}(x)\right| & \leqq\left|f(x)-G_{0}(x)\right|+\left|G_{0}(x)-H_{\delta}(x)\right| \\
& <e\left(G_{0}\right)-\eta+\eta=e\left(G_{0}\right) .
\end{aligned}
$$

Combining this inequality and the previous one for $x \in U$, we have

$$
\left|E\left(H_{\delta}, x\right)\right|<e\left(G_{0}\right), \quad x \in X=U \cup V .
$$

and $G_{0}$ is not best, proving necessity. The theorem is proven.

Let us suppose that $E\left(G_{0}, x\right) \cdot\left(G_{1}(x)-G_{0}(x)\right)>0$ for all $x \in M\left(G_{0}\right)$ and $\left\{H_{\lambda}\right\}$ is a $\lambda$-set for $G_{0}$ and $G_{1}$. For $\lambda$ sufficiently small, $\left|E\left(H_{\lambda}, x\right)\right|<e\left(G_{0}\right)$ for all $x \in M\left(G_{0}\right)$. We then apply Theorem 1 to get

Corollary. Let $\mathscr{G}$ have the betweeness property. An element $G_{0}$ of $\mathscr{G}$ is a best approximation if and only if there exists no element $G_{1} \in \mathscr{G}$ such that $E\left(G_{0}, x\right)$ $\cdot\left(G_{1}(x)-G_{0}(x)\right)>0$ for all $x \in M\left(G_{0}\right)$.

4. An error determining set on which best approximations agree. Let $\mathscr{G}^{*}$ be the set of best approximations to $f$ and $N=\bigcap M(G), G \in \mathscr{G}^{*}$. We will show in this section that if $\mathscr{G}^{*}$ is nonempty then $N$ is nonempty, best approximations must agree on $N$ and that $N$ is an error determining set, that is, there exists no approximant $F$ such that $|E(F, x)|<\inf \{e(G): G \in \mathscr{G}\}$ for $x \in N$. In the cases of approximation by linear or rational families of finite dimension, it can easily be shown that if $\mathscr{G}^{*}$ is nonempty, there exists an element $F \in \mathscr{G}$ such that $M(F)=N$; in the linear case any element of the convex interior of $\mathscr{G}^{*}$ is such an $F$. This is not true in general, for let $X=[0,1]$ and $\mathscr{G}$ be the set of monotonic continuous functions $G$ with $G$ zero in a neighborhood of the point zero. In the approximation of $f=1$ any element $G$ of $\mathscr{G}$ such that $\|1-G\|=1$ is a best approximation and $N=\{0\}$, but there is no element $G$ such that $M(G)=N$.

Lemma 3. Let $\mathscr{G}$ have the betweeness property and $\mathscr{G}^{*}$ be nonempty. Given a finite number $G_{1}, \ldots, G_{n}$ of elements of $\mathscr{G}^{*}$ there exists an element $G_{0}$ of $\mathscr{G}^{*}$ such that $\bigcap_{k=1}^{n} M\left(G_{k}\right) \supset M\left(G_{0}\right)$.

Proof. Let $G_{1}$ and $G_{2}$ be any two best approximations and $\bar{G}_{1}$ be any element of the $\lambda$-set corresponding to $G_{1}$ and $G_{2}, 0<\lambda<1$, then for all $x \in X, \bar{G}_{1}(x)$ lies between $G_{1}(x)$ and $G_{2}(x)$,

$$
\left|E\left(\bar{G}_{1}, x\right)\right| \leqq \sup \left\{\left|E\left(G_{1}, x\right)\right|,\left|E\left(G_{2}, x\right)\right|\right\}
$$

with equality only if $G_{1}(x)=G_{2}(x)$. It follows that $\bar{G}_{1}$ is a best approximation and $M\left(\bar{G}_{1}\right) \subset M\left(G_{1}\right) \cap M\left(G_{2}\right)$. Similarly, there exists $\bar{G}_{k} \in \mathscr{G}^{*}$ such that $\bar{G}_{k}$ is in the $\lambda$-set corresponding to $\bar{G}_{k-1}$ and $G_{k+1}, 0<\lambda<1$, and $M\left(\bar{G}_{k}\right) \subset \bigcap_{j=1}^{k+1} M\left(G_{j}\right)$, $k=2, \ldots, n-1$. The required approximant in $\mathscr{G}^{*}$ is $\bar{G}_{n-1}$ and the lemma is proven.

Corollary. Let $G_{0}, G_{1} \in G^{*}$, then the $\lambda$-set $\left\{H_{\lambda}\right\}$ for $G_{0}$ and $G_{1}$ is contained in $\mathscr{G}^{*}$. 
LEMMA 4. Let $\mathscr{G}$ have the betweeness property. If $\mathscr{G}^{*}$ is nonempty, $N$ is nonempty.

Proof. If $N$, an intersection of a nonempty family of closed sets, were empty, it could be expressed as a finite intersection of these sets.

$$
N=\bigcap_{k=1}^{n} M\left(G_{k}\right), \quad G_{k} \in \mathscr{G}^{*} .
$$

By the previous lemma there exists $G_{0} \in \mathscr{G}^{*}$ such that $\bigcap_{k=1}^{n} M\left(G_{k}\right) \supset M\left(G_{0}\right)$. It follows from the definition of $N$ that $N=M\left(G_{0}\right)$. But $M\left(G_{0}\right)$ is nonempty and so we have a contradiction proving the lemma.

LEMMA 5. Let the family $\mathscr{G}$ of real continuous functions have the betweeness property. Let $G_{0}, G_{1} \in \mathscr{G}^{*}$, then $G_{0}(x)=G_{1}(x)$ for all $x \in N$.

Proof. Let $G_{0}, G_{1} \in \mathscr{G}^{*}$ be given and select a $\lambda$-set $\left\{H_{\lambda}\right\}$ corresponding to $G_{0}$ and $G_{1}, 0<\lambda<1$. If $G_{0}(x) \neq G_{1}(x)$ for some $x$, then

$$
\left|E\left(H_{\lambda}, x\right)\right|<\max \left\{\left|E\left(G_{0}, x\right)\right|,\left|E\left(G_{1}, x\right)\right|\right\}
$$

for $0<\lambda<1$ and since $\left\{H_{\lambda}\right\} \in \mathscr{G}^{*}, x \notin N$.

LEMMA 6. Let $\mathscr{G}$ have the betweeness property. If $\mathscr{G}^{*}$ is nonempty there exists no approximant $G$ such that $|E(G, x)|<\inf \{e(G): G \in \mathscr{G}\}$ for all $x \in N$.

Proof. Suppose such a $G$ exists, then by continuity of $E(G, \cdot)$, the inequality

$$
|E(G, x)|<\inf \{e(G): G \in \mathscr{G}\}=\Delta(f, \mathscr{G})
$$

holds on an open cover $U$ of $N$. Let $V=X \sim U$, then $V$ is nonempty (for otherwise $e(G)<\Delta(f, \mathscr{G})$, which is impossible).

Since

$$
\left\{\cap(V \cap M(F)): F \in \mathscr{G}^{*}\right\}=V \cap N=\varnothing
$$

is an intersection of closed sets in a compact space, there exists a finite set $G_{1}, \ldots, G_{n}$ of elements of $\mathscr{G}^{*}$ such that $\bigcap_{k=1}^{n}\left(V \cap M\left(G_{k}\right)\right)=\varnothing$. Applying Lemma 3, there exists $G_{0} \in \mathscr{G}^{*}$ such that $M\left(G_{0}\right) \subset \bigcap_{k=1}^{n} M\left(G_{k}\right) \subset U$. Now let $\left\{H_{\lambda}\right\}$ be a $\lambda$-set corresponding to $G_{0}$ and $G, H_{0}=G_{0}, H_{1}=G$. Since $E\left(H_{\lambda}, x\right)$ is between $E\left(G_{0}, x\right)$ and $E(G, x)$ for $0<\lambda<1$ and $x \in U$,

$$
E\left(H_{\lambda}, x\right)<e\left(G_{0}\right), \quad 0<\lambda<1, x \in U .
$$

Now let $\eta=e\left(G_{0}\right)-\sup \left\{\left|E\left(G_{0}, x\right)\right|: x \in V\right\}$. As the sequence $\left\{H_{1 / k}\right\}$ converges uniformly to $G_{0}$, there exists $\delta>0$ such that $\left\|G_{0}-H_{\delta}\right\|<\eta$. For $x \in V$ we have

$$
\begin{aligned}
\left|E\left(H_{\delta}, x\right)\right| & =\left|f(x)-H_{\delta}(x)\right| \\
& \leqq\left|f(x)-G_{0}(x)\right|+\left|G_{0}(x)-H_{\delta}(x)\right|<e\left(G_{0}\right)-\eta+\eta=e\left(G_{0}\right) .
\end{aligned}
$$

Combining this inequality for $x \in V$ with the earlier one for $x \in U$, we have $E\left(H_{\delta}, x\right)<e\left(G_{0}\right), x \in X$, and so

$$
e\left(H_{\delta}\right)<\inf \{e(G): G \in \mathscr{G}\} .
$$

This is a contradiction and the lemma is proven. 
5. Uniqueness results. Lemmas 5 and 6 are very powerful results. Using them we can obtain many uniqueness results. We give below the most general uniqueness result, a generalization of Haar's classical result concerning necessary and sufficient conditions for best linear approximations to be unique. After this result was obtained it was noted that it includes a uniqueness result of Singer [6] for approximation by arbitrary linear subspaces of $C(X)$.

Definition. A family $\mathscr{G}$ of real continuous functions is said to have zero-sign compatibility if for any two distinct elements $G$ and $H$, any closed subset $Z$ of the zeros of $G-H$, and any continuous function $s$ which takes the values +1 or -1 on $Z$, there exists $F \in \mathscr{G}$ such that

$$
\operatorname{sgn}(F(x)-G(x))=s(x), \quad x \in Z .
$$

Without loss of generality we can assume $\|s\|=1$.

TheOREM 2. Let $\mathscr{G}$ have the betweeness property. A necessary and sufficient condition that for every continuous function a best approximation is unique is that $\mathscr{G}$ have zero-sign compatibility.

Proof. Suppose that for two distinct elements $G$ and $H$, a closed subset $Z$ of the zeros of $G-H$, and a continuous function $s,\|s\|=1$, which takes the values +1 or -1 on $Z$, there exists no element $F$ for which (*) holds.

Define:

then

$$
f(x)=G(x)+s(x)[\|G-H\|-|G(x)-H(x)|]
$$

$$
E(G, \cdot)=s(x)[\|G-H\|-|G(x)-H(x)|] .
$$

For $x \in Z$ we have $E(G, x)=s(x)\|G-H\|$, hence $Z \subset M(G)$. If a better approximant $F$ existed it would satisfy

$$
\operatorname{sgn}(F(x)-G(x))=s(x), \quad x \in Z,
$$

which is impossible by hypothesis. Hence $G$ is a best approximation to $f$ and since

$$
\begin{aligned}
|f(x)-H(x)| & \leqq|f(x)-G(x)|+|G(x)-H(x)| \\
& \leqq\|G-H\|-|G(x)-H(x)|+|G(x)-H(x)|=\|G-H\|,
\end{aligned}
$$

$H$ is also a best approximation to $f$, proving necessity.

REMARK. The proof of necessity assumes nothing about $\mathscr{G}$ and therefore shows that zero-sign compatibility is necessary for uniqueness, $\mathscr{G}$ any approximating family.

Suppose now that $\mathscr{G}$ has zero-sign compatibility and $G, G_{1}$ are distinct best approximations. Therefore $G(x)=G_{1}(x)$ for $x \in N$ by Lemma 5 . Let the function $s$ be $E(G, \cdot) /\|E(G, \cdot)\|$ then by zero-sign compatibility there exists an element $F$ such that $\operatorname{sgn}(F(x)-G(x))=\operatorname{sgn}(E(G, x))$ for $x \in N$. Let $\left\{H_{\lambda}\right\}$ be a $\lambda$-set for $G$ and $F, H_{0}=G, H_{1}=F$. The sequence $\left\{H_{1 / k}\right\}$ converges uniformly to $G$ so for some $\delta>0$, $E\left(H_{\delta}, x\right)$ will be between $E(G, x)$ and $-E(G, x)$ for all $x \in N$, hence $\left|E\left(H_{\delta}, x\right)\right|$ $<|E(G, x)|=e(G)$ for all $x \in N$. This contradicts Lemma 6 so sufficiency is proven. 
We now consider approximation on a compact subset $Y$ of $X$. If the betweeness property holds on $X$, it holds on $Y$.

LeMmA 7. Let $X$ be a compact normal space and $Y$ a compact subset of $X$. If $\mathscr{G}$ has zero-sign compatibility on $X, \mathscr{G}$ has zero-sign compatibility on $Y$.

Proof. Let $(G, H)$ be a pair of distinct elements of $\mathscr{G}$. Let $Z$ be a closed subset of $Y \cap\{x: G(x)-H(x)=0\}$ then $Z$ is a closed subset in $X$ of the zeros of $G-H$. Let $s^{\prime}$ be a continuous mapping of $Y$ into $[-1,1]$ taking values +1 or -1 on $Z$. Since $X$ is a normal space, there exists by the Tietze extension theorem $s \in C(X),\|s\|=1$, $s(x)=s^{\prime}(x)$ for $x \in Y$. Let $\mathscr{G}$ have zero-sign compatibility on $X$; then there exists $F \in \mathscr{G}$ such that

$$
\operatorname{sgn}(F(x)-G(x))=s(x)=s^{\prime}(x), \quad x \in Z .
$$

From the lemma and Theorem 2 we obtain

Corollary. Let $X$ be a compact normal space. Let $\mathscr{G}$ have the betweeness property and best approximations on $X$ to any continuous function be unique, then best approximations on any compact subset of $X$ are unique to any continuous function.

We now consider approximation by an open subset $\mathscr{G}^{\prime}$ of $\mathscr{G}$. If $\mathscr{G}$ has the betweeness property, it follows that the function $F$ in the definition of zero-sign compatibility can be chosen arbitrarily close to the function $G$ of that definition. If $G \in \mathscr{G}^{\prime}$ it follows that $F$ can be chosen such that $F \in \mathscr{G}^{\prime}$. It follows that if $\mathscr{G}$ has zero-sign compatibility, so does $\mathscr{G}^{\prime}$. We obtain:

Corollary. Let both $\mathscr{G}$ and $\mathscr{G}^{\prime}$, an open subset of $\mathscr{G}$, have the betweeness property. If every continuous function has at most one best approximation from $\mathscr{G}$, every continuous function has at most one best approximation from $\mathscr{G}^{\prime}$.

Less general but simpler uniqueness results can be developed in terms of the sign changing property and property $Z$.

Definition. $\mathscr{G}$ has the sign changing property of degree $n$ at $G$ if for any $n$ distinct points $\left\{x_{1}, \ldots, x_{n}\right\}$ and $n$ real numbers $w_{1}, \ldots, w_{n}$ which are either +1 or -1 , there exists an approximant $F$ such that

$$
\operatorname{sgn}\left(F\left(x_{k}\right)-G\left(x_{k}\right)\right)=w_{k}, \quad k=1, \ldots, n .
$$

We need not specify the closeness of $F$ to $G$ in the above definition since if such an $F$ exists, there exists with the betweeness property such an $F$ arbitrarily close to $G$.

Definition. $\mathscr{G}$ has property $Z$ of degree $n$ at $G$ if $G-F$ having $n$ zeros implies $F=G$.

Let $\mathscr{G}$ have the betweeness property. The $F$ in the definition of the sign changing property can be chosen such that for given $\varepsilon>0,\|F-G\|<\varepsilon$. Let $G \in \mathscr{G}^{*}$. If $\mathscr{G}$ has the sign changing property of degree $n$ at $G$ then $G$ either coincides with the function $f$ being approximated or $N$ has at least $n+1$ points, for if it had less we could find $F$ 
such that $|E(F, x)|<e(G)$ for $x \in N(X)$, which contradicts Lemma 6. If $\mathscr{G}$ has property $Z$ of degree $n$ at $G$ then by Lemma 5 best approximations must be identical if $N$ has $n$ or more points. We therefore have:

THEOREM 3. Let $\mathscr{G}$ have the betweeness property and $G \in \mathscr{G}^{*}$. If $\mathscr{G}$ has property $Z$ of degree $n+1$ at $G$ and the sign changing property of degree $n$ at $G$ then $G$ is a unique best approximation.

\section{BIBLIOGRAPHY}

1. B. Brosowski, Über die Eindeutigkeit der rationalen Tschebyscheff Approximationen, Numer. Math. 7 (1965), 176-186.

2. E. W. Cheney, Introduction to approximation theory, McGraw-Hill, New York, 1966.

3. E. W. Cheney and H. L. Loeb, Generalized rational approximation, SIAM J. Numer. Anal. 1 (1964), 11-25.

4. G. Meinardus and D. Schwedt, Nicht-lineare Approximation, Arch. Rational Mech. Anal. 9 (1964), 329-351.

5. John Rice, Tchebycheff approximation in several variables, Trans. Amer. Math. Soc. 109 (1963), 444-466.

6. Ivan Singer, On best approximation of continuous functions, Math. Ann. 140 (1960), 165-168.

UNIVERSITY OF WESTERN ONTARIO, LONDON, ONTARIO, CANADA 